\title{
Soft actuation and sensing towards robot-assisted facial rehabilitation
}

\author{
Amir Firouzeh ${ }^{1}$ and Jamie Paik ${ }^{1}$
}

\begin{abstract}
Continuing research efforts in robot-assisted rehabilitation demand more adaptable and inherently soft wearable devices. A wearable rehabilitative device is required to follow the motion of the body and to provide assistive or corrective motions to restore natural movements. Providing the required level of fluidity in wearable devices becomes a challenge for rehabilitation of more sensitive and fragile body parts, such as the face. To address this challenge, we propose a soft actuation method based on a tendon-driven robotic origami (robogami) and a soft sensing method based on a strain gauge with customized stretchable mesh design. The proposed actuation and sensing methods are compatible with the requirements in a facial rehabilitative device.

The conformity of robogamis originates from their multiple and redundant degrees of freedom and the controllability of the joint stiffness, which is provided by adjusting the elasticity modulus of an embedded shape memory polymer (SMP) layer. The reconfiguration of the robogami and the trajectory and directional compliance of its end-effector are controlled by modulating the temperatures, hence the stiffness, of the SMP layers. Here we demonstrate this correlation using simulation and experimental results.

In this paper, we introduce a thin and highly compliant sensing method for measuring facial movements with a minimal effect on the natural motions. The measurements of the sensors on the healthy side can be used to calculate the required tendon displacement for replicating the natural motion on the paralyzed side of the face in patients suffering from facial palsy.
\end{abstract}

\section{INTRODUCTION}

Facial neuro-motor disorder can be caused by infections, accidents, strokes or tumors. The most common cause for facial paralysis is Bell's palsy which annually affects 20 people over 100,000 of population [1]. Physiotherapy is often prescribed as a treatment for facial palsy and studies suggest its effectiveness alongside other methods in mime therapy [2]. Often the disability affects one side of the face and the goal in rehabilitation is restoring the motion of the paralyzed side [3]. The compliant sensing and actuation methods can be utilized in a wearable device for robot-assisted rehabilitation. Such a robot provides a desired level of assistance towards regaining synchronized and symmetric motion. Also, the repeatability of the robot's motion and the feedback data on the recovery rate make it possible to perform organized studies on the effectiveness of the treatment which is a subject of ongoing studies in the rehabilitation field [4].

The novel soft technologies for actuation and sensing and the novel fabrication methods which has made the manufacturing of customized robots easy and price-effective have lead to emerging wearable soft devices for robotassisted rehabilitation [4]-[13]. In this paper, we introduce

\footnotetext{
${ }^{1}$ The authors are with Reconfigurable Robotics Laboratory, EPFL, Switzerland Jamir.paik@epfl.ch
}

a soft actuation method with compliance control and soft sensing method for future generations of wearable devices. The proposed technologies are specifically appealing for demanding tasks such as robot-assisted facial rehabilitation. The complexity of motions, the required degrees of freedom $(\mathrm{DoF})$, and the geometrical constrains are the main challenges for implementing the existing technologies in this application.

\section{A. State of the art}

Various methods of soft actuation based on different physical principles are proposed for wearable rehabilitative devices including soft pneumatic actuators [7], [12], smart materials [4], [6], and tendon driven under-actuation [10], [11], [13]. Tendon driven systems are specifically desirable in applications with demanding geometrical constraints in which smart materials can not meet the actuation requirements, i.e. band width, range of motion, and load capacity. In this research, we propose an under-actuated robotic origami, robogami, platform for soft actuation. The multiple and redundant DoFs of these robots makes them conformable and allow them to perform complex reconfigurations. The folding pattern of a polymer sheet determines the DoFs in the robogamis [14]. This allows us to design joints along different directions in the robot's plane. Moreover, rolling joints allow us to place DoFs in the direction normal to the robot's plane [15]. These rotational DoFs can be arranged in any sequence to achieve a desired reconfiguration and trajectory for the end-effector. The joints are actuated through a tendon that is routed with a desired offset from the axis of rotation. The energy distribution among DoFs is assigned by controlling the joints' stiffness. In this approach we use a single input to drive multiple DoFs and points of actuation to achieve synchronized motions. Compared to direct actuation using a tendon, as proposed in [4], [6], the under-actuated robogami platform generates more complex trajectories and provides control over the compliance of actuation in different directions.

For effective interaction, a wearable rehabilitative device is required to have adjustable compliance to support the loading requirements in different training at different stages of therapy. Different methods for adjusting the compliance of soft robots have been proposed in the literature [16][28]. Adjustable stiffness layers, ASLs, based on the stiffness variation of shape memory polymers, SMPs, are effectively used for controlling the compliance of robogamis and their mode of interaction with the environment [15], [16], [29]. Here, we use the same ASL design which was previously confirmed to provide more than 40 times stiffness variation 


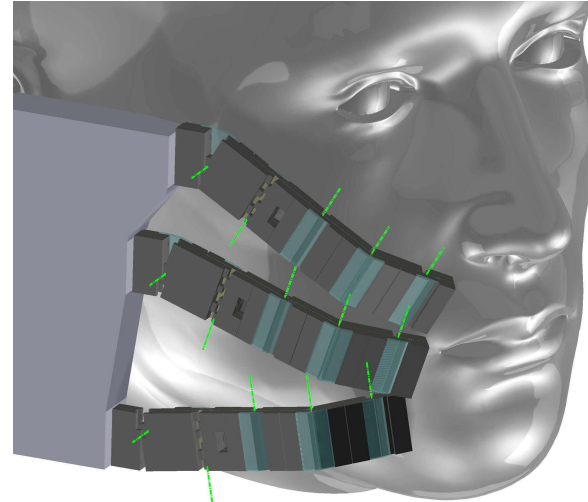

Fig. 1: The concept of a facial rehabilitative robogami. The power distribution between the joints, hence the reconfiguration of the fingers, is determined by the stiffness assigned to each joint. The initial reconfiguration of the fingers is set using the first joint, for setting the direction of actuation, and the forth joint, for coming in contact with the face. After the initial reconfiguration, these joints are locked in place and the other joints are activated for applying displacements with a desired level of compliance.

between $30{ }^{\circ} \mathrm{C}$ and $110{ }^{\circ} \mathrm{C}$ [29]. A stretchable heater embedded in the SMP allows us to control the temperature of the SMP layer and consequently the joint stiffness.

Different sensing principles are proposed in the literature for measuring the motion of the human body [8], [9], [30], [31]. Mechanical transparency, large range of motion, customizability, and reliable measurements despite the variation in ambient condition, e.g. temperature, are the main requirements from a sensing technology for a wearable rehabilitative device. The method that we use to measure facial movements is based on electrical resistance change of a metal path laminated on a polymer sheet. A pattern that is cut through the composite produces a stretchable mesh structure. The large range of motion; upto $40 \%$, mechanical transparency, and thin profile (under $100 \mu \mathrm{m}$ ) of this sensing technology make it a desirable choice for measuring facial movements. Moreover, the control over strain sensitivity [32] allows us to use gauges with opposite sensitivity for measuring displacement and to compensate the electrical resistance variation due to the change in the ambient condition.

\section{B. The concept of the facial rehabilitative robogami with sensor network}

A network of soft sensors on the healthy side of the face can measure the displacement of select points with a minimal effect on the natural motion. The sensor readings are then used to actuate a robogami with multiple points of contact on the face to restore the same motion on the affected side of the face. Fig. 1 presents an early conceptual design of a robogami with three points of actuation. Based on the desired motion, a sequence of joints for producing a $3 \mathrm{D}$ trajectory is designed. The joints with adjustable stiffness allow us to distribute the input energy from a single source between different fingers and different joints in each finger in order to produce desired trajectories for the multiple points of actuation on the face. The joint stiffness control also allows us to control the compliance of the end-effector of each finger along different directions. This soft actuation method with compliance control can be utilized in rehabilitation where synchronized motion of multiple points of actuation and many joints is required for training certain muscle groups. The multiple DoFs of robogamis allow for initial reconfiguration and activation of different actuation patterns for adapting to the needs of each patient and the requirements for a specific training. While the transition between different training modes is time consuming due to the slow nature of the heat transfer, in the order of 10 seconds, repeated actuation in each mode can be much faster.

In this paper, we study the motion of a single sequence of joints which is presented in Fig. 2. A tendon that runs along the robogami drives the joints. The joints' position and the configuration of the robot are determined by the stiffness assigned to each joint, which is controlled by adjusting the temperature of the SMP layer in each joint. We use this design in the next Section to demonstrate the initial reconfiguration and soft actuation with adjustable compliance.

The main contributions of this paper are:

- Developing a soft actuation platform with programmable actuation pattern and compliance.

- Modeling and characterizing the variable compliance of the actuation method.

- Introducing a thin and soft sensing method with high mechanical compliance for measuring facial movements.

- Confirming the applicability of the proposed methods in a preliminary prototype.

\section{UNDER-ACTUATED ROBOGAMI WITH ADJUSTABLE STIFFNESS JOINTS FOR COMPLIANT ACTUATION}

Inherently compliant robots are ideal for safe interaction with humans. The under-actuation of the multiple and redundant DoFs in tendon-driven robogamis make these robots inherently compliant and conformable. In robot-assisted rehabilitation, the compliance of the robot should be adjusted according to the training and the severity of the patient's pathology. The compliance control in robogamis is achieved through controlling the joints' stiffness which also allow us to control the trajectory of the actuation points without the need for complex actuation and control system.

In this Section, we study the compliance control of the end-effector of a simple robogami with 5 joints. Fig. 2a present the robogami design. The top layer of the first two tiles are made transparent in this figure to highlight the tendon routing. The axis of rotation for the first joint is defined by the rolling contact of the adjacent tiles. For all the rest of the joints the folding pattern of the polyimide sheet, which is defined by the glass-fiber castellated pattern, defines the axis of rotation, details of the design and manufacturing of the robogami joints are presented in [15], [29]. We use the first joint in this robot for adjusting the main direction of actuation. Using the other DoFs we apply force and displacement along the main actuation direction and control the displacement and compliance in the lateral direction Fig. 2b. The schematic in Fig. 2c presents the tendon routing, 


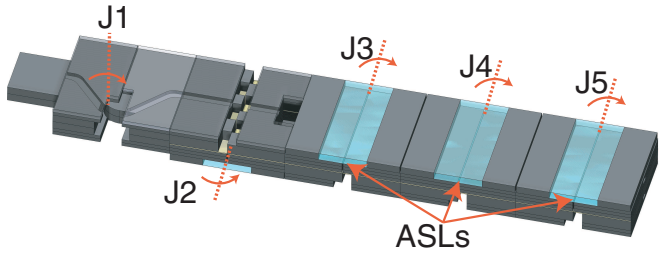

(a)

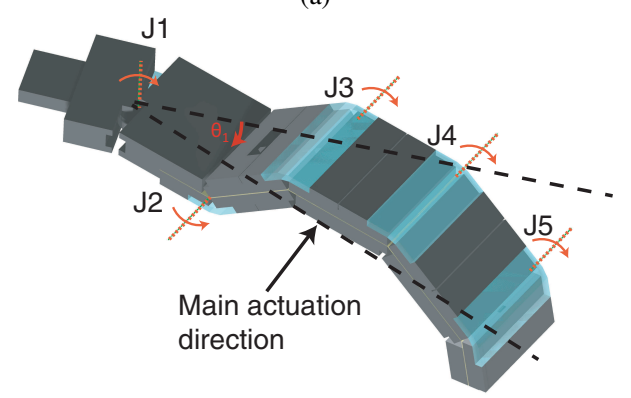

(b)

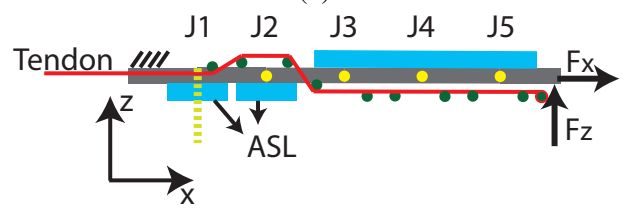

(c)

Fig. 2: The under-actuated robogami used to demonstrate soft actuation with adjustable compliance. The first joint is defined by the rolling contact point of the two adjacent tiles. All the rest of the joints are defined by the folding pattern imposed on a polyimide layer by the glass fiber castellated pattern. The top layer for the first two tiles are made transparent to highlight the tendon routing (a). The first joint allows us to orient the remaining links along a desired direction and the rest of the joints are activated at the proper temperature so that the end-effector follows a desired trajectory (b). The schematic illustrates the tendon routing. The opposite direction of motion and actuation for the second joint broadens the available compliance range for the end-effector (c). The position and the axis of rotation for the five joints, J1-J5, are presented in the schematics.

the joint axis, and the ASLs. In Section II-A, we model and simulate the motion of the last four joints in the robogami of Fig. 2 and illustrate the compliance control of the endeffector in the lateral direction. In Section II-B, we confirm the simulation results in experiment and demonstrate the soft actuation with high lateral compliance and self-adaptability.

\section{A. Model and simulation}

The trajectory of the end-effector in a tendon-driven robogami and the compliance of the robot are determined by the joints' stiffness setting. Here, we study the 2D motion of the robogami, Fig. 2, resulting from the actuation of J2-J5, design parameters for this robogami are presented in Table I. We used the design presented in [29] for the ASLs and corresponding stiffness temperature characterization results for studying the behavior of the Robogami. Using the model presented in this section and the simulation results, we can determine the proper stiffness assignment for performing a specific task.

The contact forces at the end-effector depend on the tendon tension, the configuration of the robogami, and the
TABLE I: The values of the design parameters.

\begin{tabular}{|c|c|c|}
\hline Parameters & Value & Description \\
\hline$l_{1}$ & $8.2 \mathrm{~mm}$ & $J_{1}$ and $J_{2}$ distance \\
\hline$l_{2-4}$ & $15.6 \mathrm{~mm}$ & $J_{i}$ and $J_{i+1}$ distance \\
\hline$l_{5}$ & $12.8 \mathrm{~mm}$ & Contact and $J_{5}$ distance \\
\hline$k_{A S L}$ & $108.0-2.6 \mathrm{~N} / \mathrm{mm}$ & ASL stiffness \\
\hline$h_{2}$ & $-3.25 \mathrm{~mm}$ & ASL distance from axis $J_{2}$ \\
\hline$h_{3-5}$ & $3.25 \mathrm{~mm}$ & ASL distance from axis $J_{3-5}$ \\
\hline$b_{2}$ & $-2.5 \mathrm{~mm}$ & Tendon moment arm $J_{2}$ \\
\hline$b_{3-5}$ & $3.5 \mathrm{~mm}$ & Tendon moment arm $J_{3-5}$ \\
\hline$g$ & $4 \mathrm{~mm}$ & Tendon length between the tiles \\
\hline
\end{tabular}

stiffness setting of the joints. We use the following equation, adopted from [33], to determine the stable configuration and contact forces at a given stiffness setting and tendon tension.

$$
F_{t}^{T} \dot{X}=W_{\text {contact }}+W_{A S L}
$$

This equation states the equality of the input work, the left side, with the sum of the required work for changing the configuration of the robogami, $W_{A S L}$, and the work for moving the end-effector against the contact forces, $W_{\text {contact }}$. In (1), the elements of $F_{t}$ are the tension in the tendon at each joint. Here, we neglected the tendon friction and considered constant tendon tension for all joints. The elements of $\dot{X}$ are the rate of the tendon displacement caused by the motion of each joint. To calculate the contact work, we multiply the contact forces in $\mathrm{x}$ and $\mathrm{z}$ directions, $F_{x}$ and $F_{z}$ respectively, by the rate of displacement of the contact point along these directions:

$$
W_{\text {contact }}=\left[F_{x} F_{z}\right] J \dot{\theta}
$$

$J$ relates the motion of the end-effector to the rotation of the joints, $\dot{\theta}$, and is calculated as:

$$
J=\left[\begin{array}{lll}
\sum_{j=2}^{n} l_{j} \sin \left(\sum_{i=2}^{j} \theta i\right) & \ldots & l_{n} \sin \left(\sum_{i=2}^{n} \theta_{i}\right) \\
\sum_{j=2}^{n} l_{j} \cos \left(\sum_{i=2}^{j} \theta i\right) & \ldots & l_{n} \cos \left(\sum_{i=2}^{n} \theta_{i}\right)
\end{array}\right]
$$

The rate of the tendon displacement, $\dot{X}$ in (1), has the following relation with the angular velocities of the joints:

$$
\dot{X}=T \dot{\theta}=\left[\begin{array}{cccc}
\frac{\partial X_{2}}{\partial \theta_{2}} & \ldots & 0 & 0 \\
0 & \frac{\partial X_{3}}{\partial \theta_{3}} & \ldots & 0 \\
: & : & : & \vdots \\
0 & 0 & \ldots & \frac{\partial X_{5}}{\partial \theta_{5}}
\end{array}\right] \dot{\theta}
$$

$\frac{\partial X_{i}}{\partial \theta_{i}}$, in (4), is the transmission ratio between the input and the $i^{\text {th }}$ joint. For the correlation between the rate of the joint angle change and the rate of tendon displacement for each joint, we have:

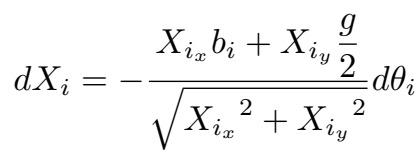



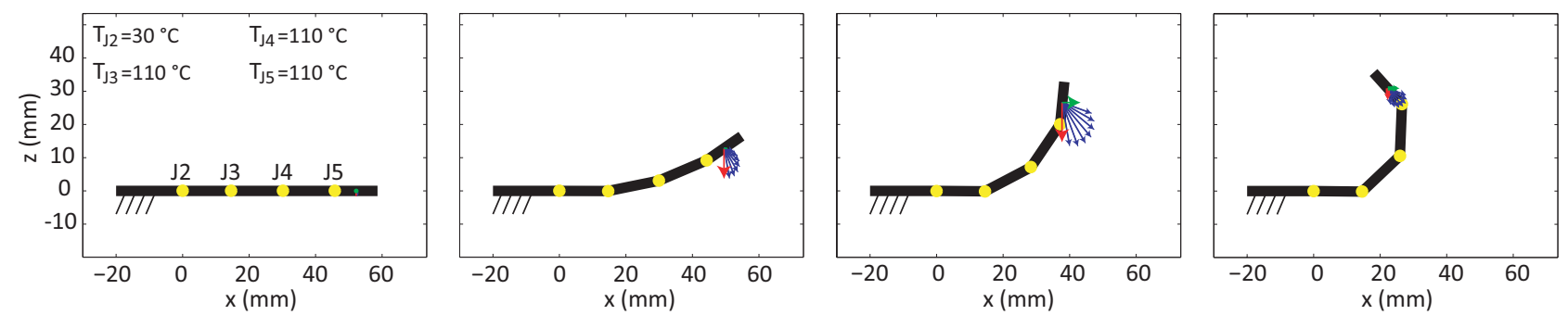

(a)
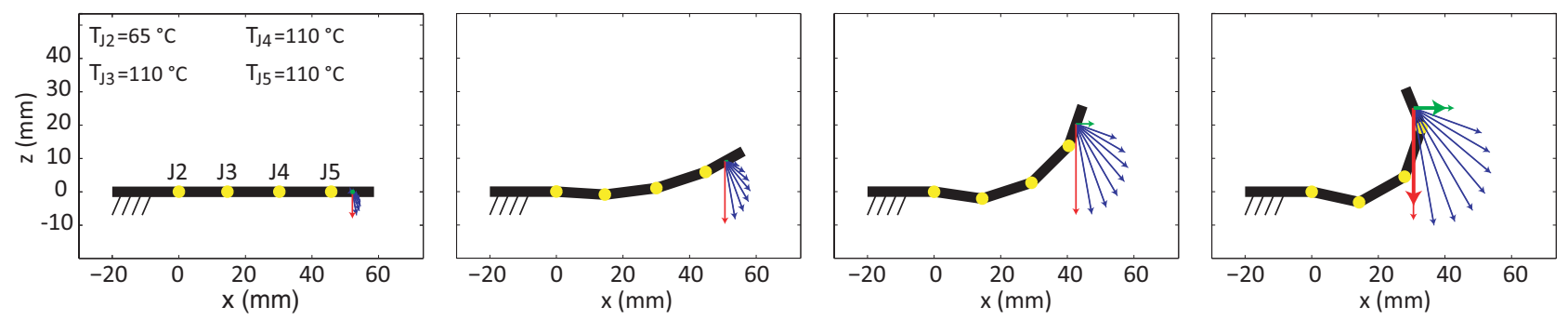

(b)

Fig. 3: The compliance of the end-effector along different directions. The arrows at the tip represent the projection of the end-effector's displacement along the direction of applied force. For each direction, $1.57 \mathrm{~N}$ (coresponding to $160 \mathrm{gr}$ ) was applied at the end-effector. As expected the compliance is dependent on the configuration of the robogami. For each stiffness setting, the compliance is reported at four configurations. In the first case, in which the last three joints are active at $110{ }^{\circ} C$, the robogami has small compliance in different directions (a). Activating the second joint at $65^{\circ} C$ increases the compliance significantly (b). The thicker arrows in the last plot represent the experimental results, which are provided for comparison.

in which, $X_{i_{x}}$ and $X_{i_{y}}$ are solved as:

$$
\begin{aligned}
& X_{i_{x}}=\frac{g}{2}\left(1+\cos \theta_{i}\right)-b_{i} \sin \theta_{i} \\
& X_{i_{y}}=\frac{g}{2}\left(\sin \theta_{i}\right)+b_{i} \cos \theta_{i}-b_{i}
\end{aligned}
$$

The required work for changing the configuration of the robogami, $W_{A S L}$, depends on the stiffness assigned to the joints and the configuration of the robogami and is calculated as:

$$
W_{A S L}=F_{A S L}{ }^{T} \dot{\Delta}=\left(\left[\begin{array}{cccc}
k_{1} & 0 & \ldots & 0 \\
0 & k_{2} & \ldots & 0 \\
: & : & : & : \\
0 & 0 & \ldots & k_{5}
\end{array}\right] \Delta\right)^{T} \dot{\Delta}
$$

The elements of $\Delta$ and $F_{A S L}$ in (8) are the elongation and the force of the ASLs, respectively. $k_{i}$ in this equation represent the stiffness of the ASL of the corresponding joint which depends on the temperature of the SMP layer. By adjusting the temperature using the embedded heater in SMP, the compliance of the joints is increased upto 40 times as the temperature increases from $30{ }^{\circ} \mathrm{C}$ to $110{ }^{\circ} \mathrm{C}$ [29].

Replacing (2) and (8) in (1) yields the following equation which correlates the tension in the tendon with the joint angles and their stiffness:

$$
F_{t}^{T} T=f^{T} J+\left(h+\frac{t_{A S L}}{2}\right)^{2}\left(K_{A S L} \sin \theta\right)^{T}
$$

In which $K_{A S L}$ represents the matrix of joints' stiffness. Using (9), we can determine the proper stiffness setting for following a desired trajectory with desired compliance

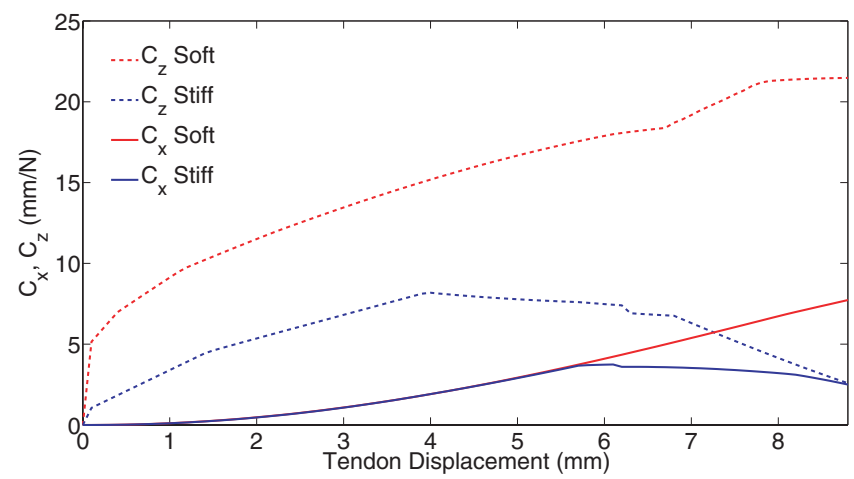

Fig. 4: The compliance of the end-effector in the main direction of actuation, $\mathrm{x}$, is comparable in both cases, stiff and soft modes, but the lateral compliance, along $\mathrm{z}$, is significantly higher in the second case. In the soft mode, the low compliance along $\mathrm{x}$ direction is desirable since it allows us to apply large forces in this direction. In $\mathrm{z}$ direction, however, higher compliance is desirable so that the robot can adjust its lateral position based on the actuation constraints.

along different directions. Fig. 3 presents the stable configuration of the robogami at different tendon displacements and temperature settings. The arrows at the end-effector represent its displacement along the direction of $1.57 \mathrm{~N}$ (corresponding to $160 \mathrm{gr}$ ) contact force which provide a measure of the compliance along different directions. In the first case, Fig. 3a, J2 is at room temperature and the stiffness of J3-J5 are set to the lowest, $110{ }^{\circ} \mathrm{C}$. The exceptionally low compliance in the first and last configurations is the result of joints being at their limits, $0^{\circ}$ and $45^{\circ}$. In the second case, Fig. 3b, the second joint is also activated but at higher stiffness, $65{ }^{\circ} \mathrm{C}$. The higher stiffness setting for $\mathrm{J} 2$ along with the smaller moment arm of the tendon, Table I, results in 


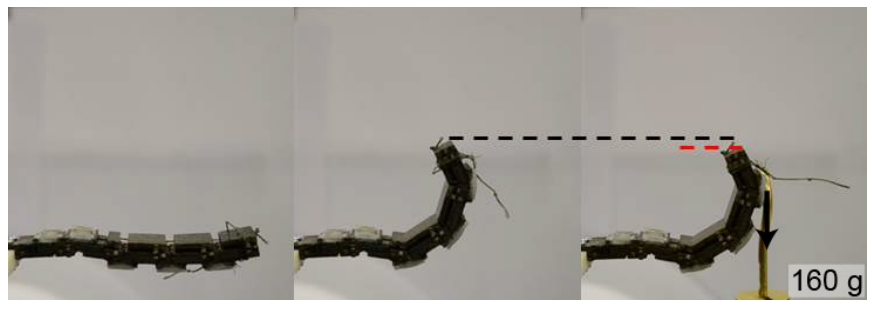

(a)

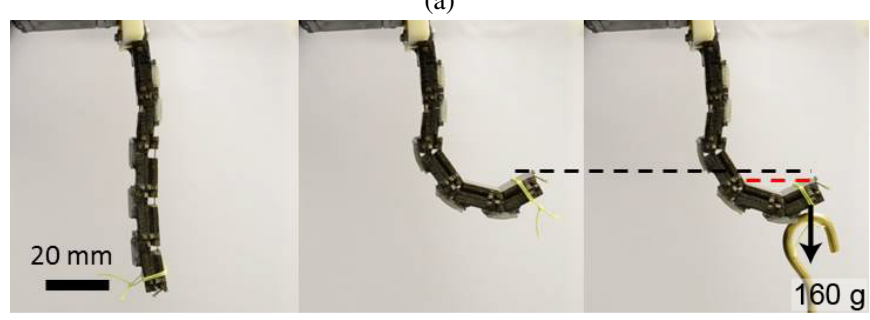

(c)

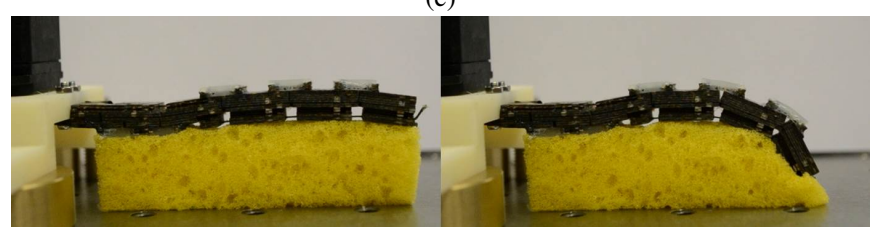

(e)

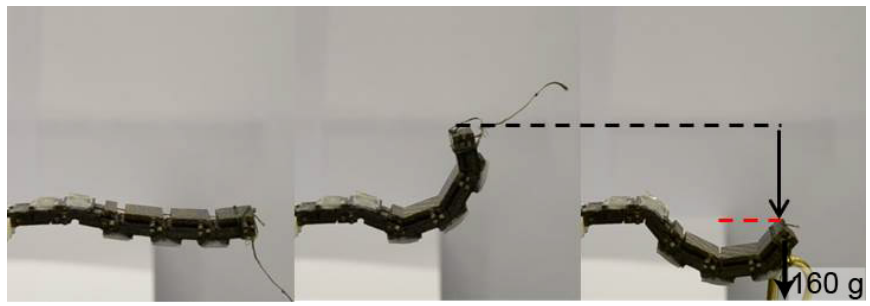

(b)

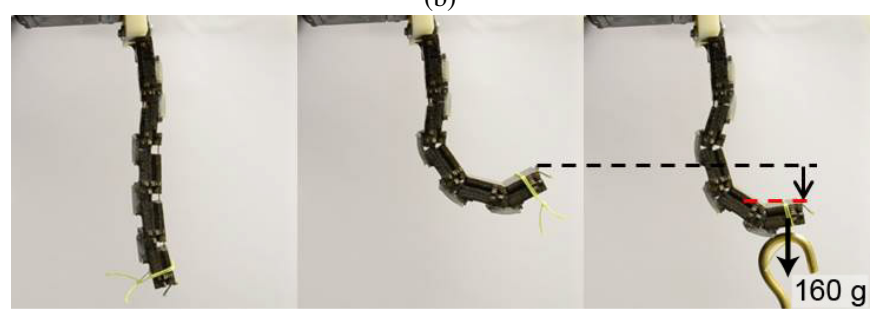

(d)

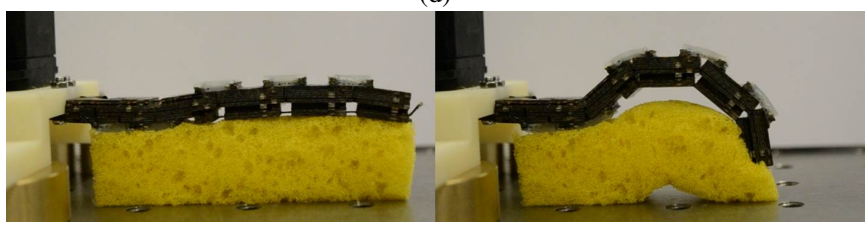

(f)

Fig. 5: Compliance control in the tendon-driven robogami. To verify the simulation results, we compared the configuration of the robogami at different stiffness settings and loading conditions. We considered loading along the main direction of actuation and the lateral direction. In the stiff mode, the end-effector has low compliance in both directions (a,c). In the soft mode, the end-effector is significantly more compliant in the lateral direction (b). The compliance along the direction of the finger also increases but not to the same extent as the lateral compliance (d). In the stiff mode, the robogami has low self-adjustment capability (e). This limits the motion of the robot in the presence of constraints. Increasing the lateral compliance of the robogami, by activating $\mathbf{J} 2$, allows the end-effector to auto adjust its lateral position and increases the range of motion in the presence of the constraint (f).

similar configurations in free displacement in both cases. The compliance of the end-effector in the two cases, however, are significantly different. Activation of $\mathrm{J} 2$ makes the robogami considerably softer in the lateral direction.

Fig. 4 is another representation of the simulation results presented in Fig. 3 which plots the compliance profile of the robogami manipulator along the two directions of interest, the main actuation direction and the lateral direction. Having control over the displacement and contact force in the actuation direction requires low stiffness in this direction, for a device similar to Fig. 1. In the lateral direction, however, high compliance is required so that the robot can self-adjust the lateral position of its end-effector. The proposed design allows us to control the lateral compliance without affecting considerably the compliance along the main actuation direction as presented in Fig. 4. As discussed previously, the compliance at the end-effector depends on the stiffness assigned to the joints and the configuration of the robogami. The sudden changes in the slope of the graphs in this figure is caused by the joints reaching their limits. The free motion trajectory of the end-effector in both cases are similar. The self-adjustment of the end-effector in the lateral direction, however, is more significant in the second case. The proper compliance for the robogami depends on the required contact forces, the mechanical properties of the object in contact, and the configuration of the robot. Once the desired compliance is determined, the model presented here is used for assigning the proper joints' stiffness.

\section{B. Experimental verification}

To verify the simulation results of the previous Section, we tested the compliance along the main actuation direction and the lateral direction. We used a mass of $160 \mathrm{~g}$ to apply the force at the tip of the manipulators. Fig. 5a-5d presents the free motion of the robogami and its configuration with force applied at the end-effector. We tested the robogami with the same stiffness settings as the ones used in Fig. 3. As predicted by the simulation, the free motion trajectory of the end-effector in both cases are similar. In the first case, the configuration change under load is rather small, around $2 \mathrm{~mm}$ in each direction. The trend of the compliance change for the second case, in which the first joint is activated, is similar to the one predicted in the simulations. The lateral compliance increases significantly and the end-effector moves $29 \mathrm{~mm}$ in the direction of the force, compared to $33 \mathrm{~mm}$ predicted in the simulation. In the direction of actuation, the compliance is much lower and under load the end-effector moves $10 \mathrm{~mm}$ in the direction of force, compared to $11 \mathrm{~mm}$ predicted in the simulation.

To highlight the significance of the lateral compliance and self-adjustment, we tested the robogami in contact with a soft object, a piece of foam. The objective here is to move the foam along the main actuation direction. The first case, Fig. 5e, presents the actuation result when only the last three 
joints are active, stiff mode. The lateral constraint prevents a large displacement in this mode. As discussed before, activating the first joint at $65{ }^{\circ} \mathrm{C}$ does not change the free motion trajectory of the finger but it makes the robogami significantly more compliant in the lateral direction. In this mode, Fig. 5f, the end-effector auto adjusts its lateral position and the robogami can apply a larger displacement. Note that if the compliance is increased too much, the robogami would move along the foam without applying any contact force or displacement to the foam. The proper compliance setting depends on the mechanical properties of the object in contact and the required contact forces.

The soft actuation method, presented in this Section, is desirable for robot assisted rehabilitation based on the ease of manufacturing, compliance control, and the potential for following different 3D trajectories by activating proper set of joints. In Section IV, we study the feasibility of using this early prototype for applying forces in a desired direction. The self-adjustment of the end-effector in this actuation method, simplifies the control problem and the hardware required for the actuation.

\section{STRETCHABLE SOFT SENSOR}

Wearable robots require sensors with fast, and accurate response and a high mechanical transparency. In the case of the facial rehabilitation, the sensors should also be lightweight and thin. In this paper, we propose a novel thin stretchable sensor manufactured by laser ablation and chemical etching, the details of the manufacturing process along with the characterization and a model for the sensitivity of this sensor are presented in [32]. The stretchable pattern which is cut through the polyimide-Inconel composite is similar to the pattern used for the stretchable heaters which are embedded in SMP for controlling its temperature. The elongation of this mesh pattern translates into the bending motion of the the micro-beams as presented in Fig. 6. The neutral plane in the cross-section of the micro-beams falls between the metal layer and the poylimide layer. Given the opposing bending direction, the metal layer is in tension in parts of the pattern and in compression in other parts. If the metal path has similar width, hence resistance, both in tension and compression, the case for the middle section in Fig. 6, there would be no significant electrical resistance change. By modulating the width in different sections of the mesh structure, we can amplify the influence of either the part in tension or compression on the electrical resistance and make the sensor with either positive or negative sensitivity. We used this feature to design a stretchable sensor with two gauges: one with positive and another with negative sensitivity as presented in Fig. 6. The differential reading of these two gauges is insensitive to environment condition such as temperature and provides reliable estimation of the elongation.

When stretched, the micro-beams that construct the mesh structure twist out of plane and bend around the axis with the minimum moment of inertia, which requires less energy compared to in plane bending. The twist direction determines the bending direction of the micro-beams. For the mesh structure with symmetric metal layer, similar to the middle part, the twist direction is random. For the parts with asymmetric metal pattern, however, the twist direction is not random and it always happens from the flat state toward the axis of minimum inertia as presented in Fig. 6. In the proposed design, the micro-beams of the top gauge are designed to twist in the direction that puts the narrow metal section in tension resulting in positive sensitivity for the gauge. The opposite twist direction of the bottom gauge, on the other hand, puts the narrow metal section in compression resulting in negative sensitivity for this gauge.

A network of sensing elements with different sensitivities based on the proposed principle can be used in wearable devices for measuring body motions. The thin profile of the sensors and their high mechanical transparency make
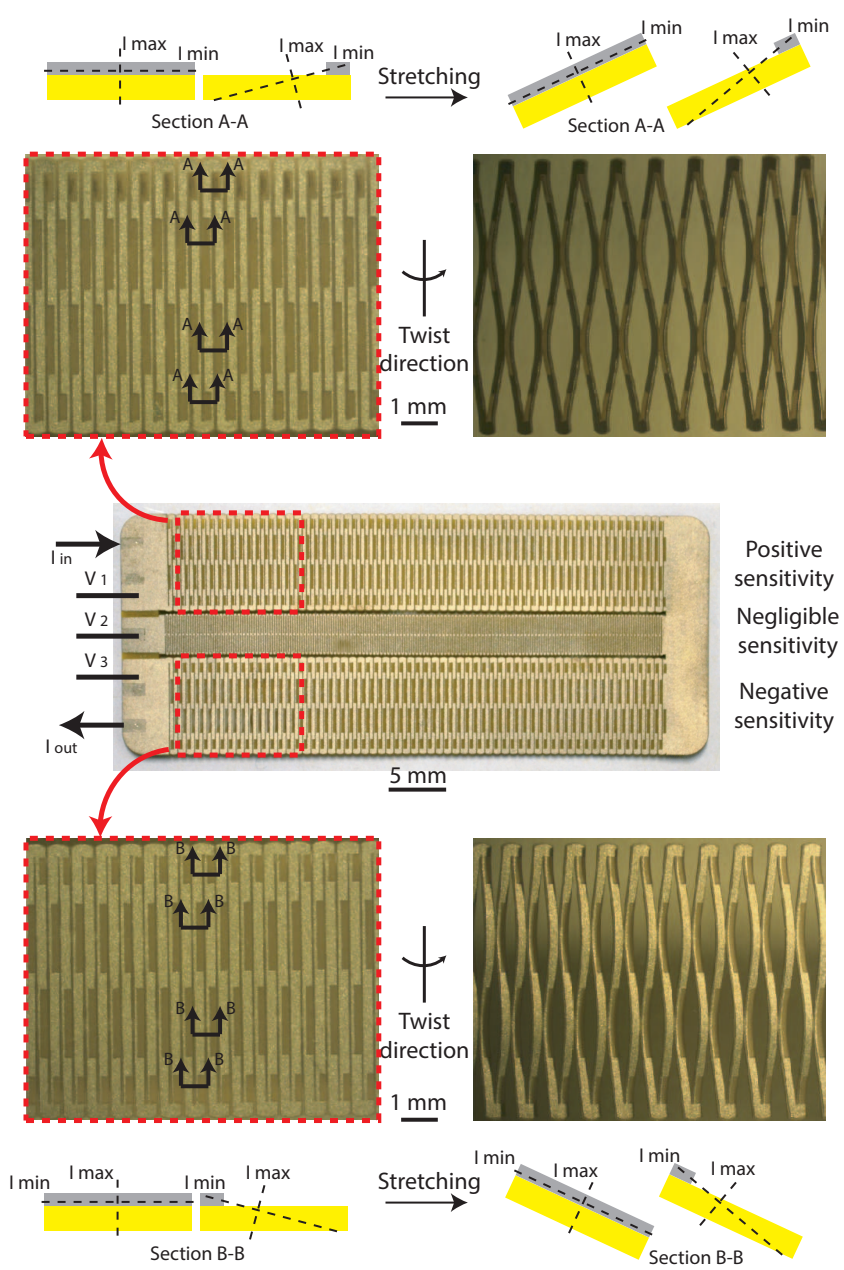

Fig. 6: A stretchable sensor based on the resistance variation of a metal film under strain. Gray and yellow in the schematics represent the metal and polyimide layers, respectively. This sensor has three distinctive sections with: positive, negative and no sensitivity to elongation. By controlling the twist direction and modulating the width of the electrical path, we can control the resistance change when the sensor is stretched. In the current design the two sensitive parts of the sensor have opposite twist directions and sensitivity to stretch. This allows us to cancel out any resistance change due to ambient temperature variation. The middle section has almost constant resistance under stretch and is used to read the voltage of the intersection of the two gauges. 
them specifically appealing for the demanding applications such as measuring facial movements. In the next Section, we illustrate the feasibility of using a single sensor, similar to the one presented in Fig. 6, for measuring the relative displacement of two points on the face.

\section{SOFT SENSING AND ACTUATION FOR FACIAL REHABILITATION}

In this Section, we present the results of preliminary tests to illustrate the applicability of the actuation and sensing methods in assistive devices for rehabilitation. Fig. 7a presents the initial reconfiguration of a simple robogami for aligning the main actuation motion with the desired direction. Fig. $7 b$ presents soft actuation of the face along this direction. For actuation, the fist joint is locked in place and the other four joints are activated with the same setting as the one used in Fig. 5f. Multiple points of actuation with more complex sequence of joints, capable of following 3D trajectories and compliance setting, will be studied in future for recreating natural motions of the face.

To demonstrate the mechanical transparency and the feasibility of measuring the relative motion of points on the face, we placed a sensor to detect and measure the displacement of a point of interest in smiling motion. We used the sensor reading in driving the robogami as presented in Fig. 8. In future, a network of similar sensors can be used for detecting the motion on the healthy side of the face and driving the rehabilitative device on the paralyzed side.

\section{CONCLUSion}

The emerging soft actuation and sensing methods are transforming the hardware used in robot-assisted rehabili-
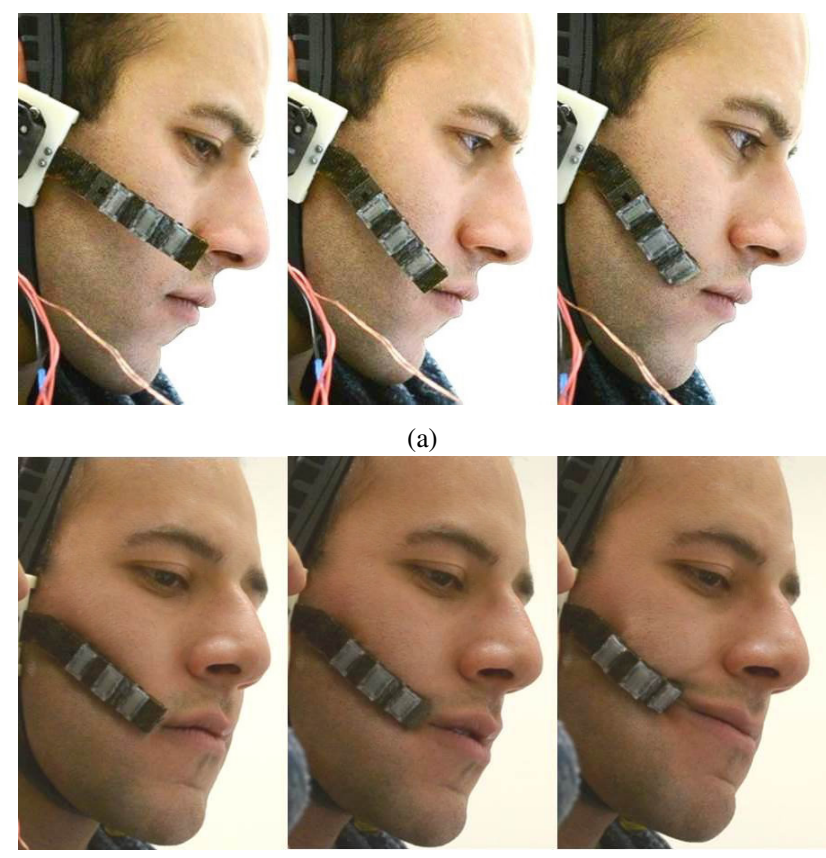

(b)

Fig. 7: Preliminary experiments using a single robogami finger for performing the initial reconfiguration (a) and soft actuation (b) on the face.

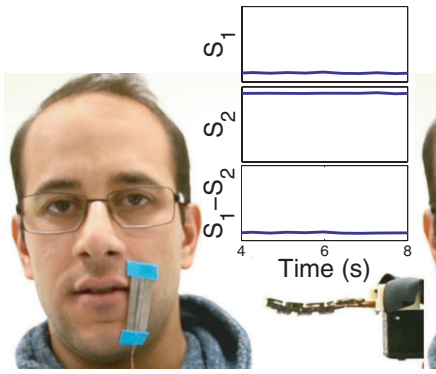

(a)

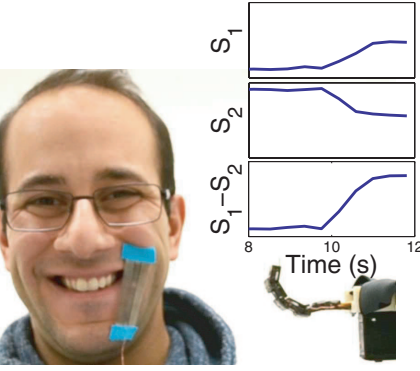

(b)
Fig. 8: Demonstrating the coupling of the soft stretchable sensor and the robogami finger. The sensor measures the displacement of the point of interest on the face and the robogami finger is actuated accordingly. (a) and (b) presents the sensor reading and the actuation of the robogami in two states. The readings from the two stretchable sensors and the subtracted value are also presented in the figure.

tation from stationary and bulky systems into wearable and light weight robots. The inherent safety, ease of control, and customizability of the novel methods of actuation can be used in complex tasks such as rehabilitation of the face. In this paper, we presented soft actuation and sensing methods for eventual application in wearable assistive devices. For the actuation, we used tendon-driven robogami platform with thermally controlled adjustable stiffness joints. The layerby-layer manufacturing of the robogamis along with the precise $2 \mathrm{D}$ fabrication methods allow us to design and manufacture robots with multiple DoFs and different embedded functions capable of complex reconfiguration. Moreover, the compliance of the end-effector can be adjusted in different directions to achieve a desired level of self-adjustment. Here, we illustrated compliance control of the end-effector of a robogami consisting of a rather simple arrangement of the joints. This method of actuation can eventually be used for applying force and displacement along a trajectory, main actuation trajectory, while maintaining a desired level of compliance along the other directions. Multiple points of actuation in robogamis can be driven using a single source to achieve a synchronized motion. The inherent softness, the ease of actuation, and the low profile of the tendon-driven robogamis make this family of soft robots an attractive choice for light-weight wearable assistive devices with multiple DoFs.

For sensing, we proposed a method based on strain induced electrical resistance change in a stretchable mesh structure. The thin profile, high mechanical transparency, and controllable sensitivity make the proposed sensing method ideal for designing a network of sensing elements for measuring relative motion of points on the face. By coupling gauges with positive and negative sensitivity, we can cancel out the effect of ambient condition on the sensor reading for more accurate estimation of the motion. We briefly studied the applicability of the sensing methods by measuring the relative displacement of two points on the face when smiling and demonstrated the feasibility of using the soft actuation method for applying large enough forces to make comparable motions. 
The next steps in this research include the study of the motion of the face for designing the required trajectories in rehabilitation and characterizing the mechanical properties of the facial muscles for estimating the required forces. Using these a robogami platform with proper number of actuation points and joint sequence will be designed to reproduce the desired trajectories and contact forces.

\section{ACKNOWLEDGMENTS}

This work was supported by Swiss National Center for Competence in Research (NCCR) in Robotics.

\section{REFERENCES}

[1] N. J. Holland and G. M. Weiner, "Recent developments in Bell's palsy," British Med. J., vol. 329, no. 7465, p. 553, 2004.

[2] C. H. Beurskens and P. G. Heymans, "Positive effects of mime therapy on sequelae of facial paralysis: stiffness, lip mobility, and social and physical aspects of facial disability," Otology \& Neurotology, vol. 24, no. 4, pp. 677-681, 2003.

[3] I. G. Williamson and T. R. Whelan, "The clinical problem of Bell's palsy: is treatment with steroids effective?" British J. General Practice, vol. 46, no. 413, pp. 743-747, 1996.

[4] D. Jayatilake, T. Isezaki, Y. Teramoto, K. Eguchi, and K. Suzuki, "Robot Assisted Physiotherapy to Support Rehabilitation of Facial Paralysis," IEEE Transactions on Neural Systems and Rehabilitation Engineering, vol. 22, no. 3, pp. 644-653, May 2014.

[5] N. Chia Bejarano, S. Maggioni, L. De Rijcke, C. A. Cifuentes, and D. J. Reinkensmeyer, "Robot-Assisted Rehabilitation Therapy: Recovery Mechanisms and Their Implications for Machine Design," pp. 197-223, 2016.

[6] D. Jayatilake, A. Gruebler, and K. Suzuki, "An Analysis of Facial Morphology for the Robot Assisted Smile Recovery," in 2008 4th International Conference on Information and Automation for Sustainability, Dec 2008, pp. 395-400.

[7] P. Polygerinos, Z. Wang, K. C. Galloway, R. J. Wood, and C. J. Walsh, "Soft robotic glove for combined assistance and at-home rehabilitation," Robotics and Autonomous Systems, vol. 73, pp. 135 - 143, 2015, wearable Robotics.

[8] N. Friedman, V. Chan, D. Zondervan, M. Bachman, and D. J. Reinkensmeyer, "MusicGlove: Motivating and quantifying hand movement rehabilitation by using functional grips to play music," in 2011 Annual International Conference of the IEEE Engineering in Medicine and Biology Society, Aug 2011, pp. 2359-2363.

[9] Y. Meng, Y. L. Park, E. Martinez-Villalpando, P. Aubin, M. Zisook, L. Stirling, R. J. Wood, and C. J. Walsh, "Soft wearable motion sensing suit for lower limb biomechanics measurements," in 2013 IEEE International Conference on Robotics and Automation, May 2013, pp. 5309-5316.

[10] C. J. Nycz, T. Btzer, O. Lambercy, J. Arata, G. S. Fischer, and R. Gassert, "Design and Characterization of a Lightweight and Fully Portable Remote Actuation System for Use With a Hand Exoskeleton," IEEE Robotics and Automation Letters, vol. 1, no. 2, pp. 976-983, July 2016.

[11] H. In, B. B. Kang, M. Sin, and K. J. Cho, "Exo-Glove: A Wearable Robot for the Hand with a Soft Tendon Routing System," IEEE Robotics Automation Magazine, vol. 22, no. 1, pp. 97-105, March 2015.

[12] M. Wehner, B. Quinlivan, P. M. Aubin, E. Martinez-Villalpando, M. Baumann, L. Stirling, K. Holt, R. Wood, and C. Walsh, "A lightweight soft exosuit for gait assistance," in 2013 IEEE International Conference on Robotics and Automation, May 2013, pp. 33623369.

[13] A. T. Asbeck, S. M. M. D. Rossi, I. Galiana, Y. Ding, and C. J. Walsh, "Stronger, Smarter, Softer: Next-Generation Wearable Robots," IEEE Robotics Automation Magazine, vol. 21, no. 4, pp. 22-33, Dec 2014.

[14] N. Doshi, B. Goldberg, R. Sahai, N. Jafferis, D. Aukes, R. J. Wood, and J. A. Paulson, "Model driven design for flexure-based Microrobots," in Proc. IEEE/RSJ Int. Conf. Int. Robot. and Sys., Sept 2015, pp. 4119-4126.

[15] A. Firouzeh and J. Paik, "An under-actuated origami gripper with adjustable stiffness joints for multiple grasp modes," Smart Materials and Structures, vol. 26, no. 5, p. 055035, 2017.
[16] A. Firouzeh, S. S. Mirrazavi Salehian, A. Billard, and J. Paik, "An under actuated robotic arm with adjustable stiffness shape memory polymer joints," in Proc. IEEE Int. Conf. Robot. and Autom., May 2015, pp. 2536-2543.

[17] M. A. McEvoy and N. Correll, "Thermoplastic variable stiffness composites with embedded, networked sensing, actuation, and control," Journal of Composite Materials, vol. 49, no. 15, pp. 1799-1808, 2015.

[18] W. Shan, T. Lu, and C. Majidi, "Soft-matter composites with electrically tunable elastic rigidity," Smart Mater. Struct., vol. 22, no. 8, p. 085005, 2013.

[19] L. Hines, V. Arabagi, and M. Sitti, "Shape Memory Polymer-Based Flexure Stiffness Control in a Miniature Flapping-Wing Robot," IEEE Tran. Robot., vol. 28, no. 4, pp. 987-990, 2012.

[20] A. Firouzeh, M. Salerno, and J. Paik, "Soft pneumatic actuator with adjustable stiffness layers for Multi-DoF Actuation," in Proc. IEEE/RSJ Int. Conf. Int. Robot. and Sys., Sept 2015, pp. 1117-1124.

[21] T. Ranzani, M. Cianchetti, G. Gerboni, I. D. Falco, and A. Menciassi, "A Soft Modular Manipulator for Minimally Invasive Surgery: Design and Characterization of a Single Module," IEEE Tran. Robot., vol. 32, no. 1, pp. 187-200, Feb 2016.

[22] A. A. Stanley and A. M. Okamura, "Controllable Surface Haptics via Particle Jamming and Pneumatics," IEEE Trans. Haptics, vol. 8, no. 1, pp. 20-30, Jan 2015.

[23] W. Ying, C. Yonghua, R. Tao, C. Qiao, Y. Changxin, Y. Yang, and L. Yingtian, "A Novel, Variable Stiffness Robotic Gripper Based on Integrated Soft Actuating and Particle Jamming ," Soft Robotics, vol. 3(3), pp. 134-143, Sep. 2016.

[24] N. G. Cheng, A. Gopinath, L. Wang, K. Iagnemma, and A. E. Hosoi, "Thermally Tunable, Self-Healing Composites for Soft Robotic Applications," Macromol. Mater. Eng., pp. n/a-n/a, 2014.

[25] W. Wang, H. Rodrigue, and S.-H. Ahn, "Deployable Soft Composite Structures," Sci. Rep., vol. 6, p. 20869, 2016.

[26] J. Shintake, B. Schubert, S. Rosset, H. Shea, and D. Floreano, "Variable stiffness actuator for soft robotics using dielectric elastomer and low-melting-point alloy," in Proc. IEEE/RSJ Int. Conf. Int. Robot. and Sys., Sept 2015, pp. 1097-1102.

[27] J. Viau, P. Chouinard, J. P. L. Bigue, G. Julio, F. Michaud, and J. S. Plante, "Tendon-Driven Manipulator Actuated by MagnetoRheological Clutches Exhibiting Both High-Power and Soft Motion Capabilities," IEEE/ASME Trans. Mech., vol. PP, no. 99, pp. 1-1, 2016.

[28] T. Takeda, Y. Shindo, and F. Narita, "Flexural stiffness variations of woven carbon fiber composite/shape memory polymer hybrid layered beams," J. Comp. Mat., vol. 49, no. 2, pp. 209-216, 2015.

[29] A. Firouzeh, M. Salerno, and J. Paik, "Stiffness control with shape memory polymer in underactuated robotic origamis," IEEE Tran. Robot., vol. PP, no. 99, pp. 1-13, 2017.

[30] T. Yamada, Y. Hayamizu, Y. Yamamoto, Y. Yomogida, A. IzadiNajafabadi, D. N. Futaba, and K. Hata, "A stretchable carbon nanotube strain sensor for human-motion detection," Nat Nano, vol. 6, no. 5, pp. 296-301, 2011, 10.1038/nnano.2011.36.

[31] B. O'Brien, T. Gisby, and I. A. Anderson, "Stretch sensors for human body motion," pp. 905618-905 618-9, 2014.

[32] A. Firouzeh and J. Paik, "The Design and Modeling of a Novel Resistive Stretch Sensor With Tunable Sensitivity," IEEE Sensors Journal, vol. 15, no. 11, pp. 6390-6398, Nov 2015.

[33] L. Birglen, T. Laliberte, and C. Gosselin, Underactuated Robotic Hands. (Springer Tracts in Advanced Robotics). Springer, 208, vol. 40 . 